\title{
Course Scheduling ACCORding to Student STRESS
}

\author{
Shuai Ma ${ }^{1}$, Ali Akgunduz ${ }^{2}$, and Yong Zeng ${ }^{{ }^{*}}$ \\ ${ }^{I}$ Concordia Institute for Information Systems Engineering, Concordia University \\ ${ }^{2}$ Department of Mechanical and Industrial Engineering, Concordia University \\ *zeng@ciise.concordia.ca
}

\begin{abstract}
As many as one in three first-year undergraduate students cannot make it back for the sophomore year. The low retention rate for students, especially engineering students, is a widespread problem. In this paper, the quantification of course difficulty and student stress is discussed, followed by a student stress model which can integrate student stress into the course scheduling problem. Some future work is presented in the conclusion.
\end{abstract}

Keywords: Course scheduling, student stress, course difficulty, cognitive measurement, quantification, retention rate.

\section{INTRODUCTION}

Engineering programs consist of a three-step training. First, students receive training with engineering fundamentals. Next, program specific topics are covered. Finally, electives or options are provided. During their first two years at university, engineering students mainly take engineering core courses and/or area core courses, which are pedagogically essential for students to be able to handle more specialized courses in their fields. Therefore students have a minimum control of their course sequences during their first two years.

It is highly common that first year engineering students have low retention rate (less than $60 \%$ in North America). Since students are admitted to engineering programs based on their high school performances, it can be presumed that they all have the academic requirements to be successful in engineering programs. However, in reality, many perform poorly despite having sufficient academic credentials in their high schools. While lack of motivation generated in freshmen classes is considered to be the main reason for low retention rate, impact of excessive workload on retention also deserves further research. It is clear that some students, particularly in their first year, are not able to cope with the workload imposed on them, which leads to an overwhelming mental stress [1].
The first year study can be regarded as a difficult task for most students in universities. The more mental resource is consumed, the more stress a student might feel. The Yerkes-Dodson Law in the arousal theory supposed that for difficult task, only an optimal arousal, or stress level, can result in the best performance, and both low and high arousal level will lead to a weak performance. Considering the low retention rate, it can be presumed that most of the students who fail to pass exams are in high stress level. In this research, due to pedagogical prerequisites, we assume that the list of first year courses and their contents may not be changed considerably. Therefore, we explore the possibility of scheduling courses in a way to reduce the student stress. In order to take the student stress in to consideration, a student stress model is proposed, which can be regarded as an objective in the course scheduling problem.

In order to quantify the student stress, the course difficulty and the students' working efficiency in daytime need to be quantified first. It is supposed by scheduling the courses with high difficulty in the time slots with high working efficiency, the student stress can be minimized. As a consequence, the new course schedule might ensure engineering students a more pleasant mental state, which hopefully can result in a higher retention rate. The quantification of student stress and the working efficiency is discussed in Section 2.

After quantifying the two necessary factors in modelling student stress, how to integrate student stress into course scheduling problem is discussed. Two methods are proposed here. The first one is a two-stage method. In the first stage, a set of feasible scheduling solutions is generated by a course scheduling system. In the second stage, these solutions are ranked by student stress, and the best one can be considered as the schedule with an optimal student stress. The second method is to integrate the student stress into the objective of the course scheduling algorithm with a penalty method, or solve the problem as a multi-objective optimization problem. How to integrate the student stress model into the course scheduling problem is discussed in Section 3. 
For the proposed resolution, some future work is discussed in Section 4.

\section{QUANTIFICAITON}

In order to evaluate feasible scheduling solutions with considering the course difficulty and the student stress, the difficulty of courses and the stress of students should be quantified first. Mathematically, two weight vectors, which describe the course difficulty and the student stress respectively, should be acquired based on some quantification method and criterion.

\subsection{Course Difficulty}

It is widely known that different courses in different universities have different difficulty. Generally speaking, the difference may result from different university requirements, professors, disciplines and the students per $s e$. Since the number of the courses are usually large, ranking the course difficulty in a university directly is usually hard. Four methods for course difficulty are introduced here: 1) pairwise comparison; 2) analytic hierarchy process; 3) GPA-based method; and 4) physiological measurement method.

Pairwise comparison method evaluates each two alternatives based on some criteria, and the better one get a higher score. Finally each alternative has a total score and the score vector can be regarded as the weight vector. This method is easy to use, but it simplifies the different magnitude of the differences with a unified score difference, and part of the difference information is lost.

Based on pairwise comparison method, the analytic hierarchy process (AHP) method evaluates multiple criterion simultaneously. There are three levels in the AHP hierarchy: goal, criteria and alternative, which refer to more easily comprehended sub-problems. Firstly the evaluation is decomposed into multiple criterion, and a priority weight vector is generated by pairwise comparison to describe the preference for the criterion. Secondly the alternatives are compared in pairs in a similar way. Finally the weighted score is computed for each alternative. This score vector can be taken as the weight vector. Comparing with the pairwise comparison method, the AHP method regards the ranking problem as a system, by decomposing the criteria and comparing the alternatives separately, a more convincing ranking result is acquired. A practical course evaluation example can be found in [2].

Two methods mentioned above need evaluations from professors and/or students, and the quantification is achieved by qualitative comparison, which will lead to a subjective result. The third method is based on grade point average (GPA). As a manifestation of academic achievement, it can be presumed that an averaged GPA score reflects the overall difficulty of a course. A detailed discussion about evaluating course difficulty with adjusting GPA can be found in [3].

Generally an academic workload could result in a mental fatigue, which can be illustrated that after an intensive class, students will probably feel tired. And this mental fatigue can trigger a higher stress level, which will impact subsequent learning activities. Mental fatigue can be reflected in physiological features, such as heart rate variability (HRV) and galvanic skin response (GSR). Cinaz et al. [4] measured HRV features to quantify the mental workload levels during office-work. Besides, additional subjective difficulty levels were rated by NASA task load index (TLX).

It is comprehensible that course difficulty quantification is more or less subjective, especially a comparison across disciplinary lines is involved. Manipulating multiple variables statistically might improve the quality of the quantification. A systematic study on quantifying course difficulty based on statistics can be found in [5].

\subsection{Working Efficiency}

The student efficiency in daytime is elusive to quantify. Here high efficiency generally refers to a good mental state to study, which is embodied in high work engagement and no overwhelming mental stress. It is also presumed that high efficiency will result in a high performance with a better chance. It was pointed out that a number of factors can affect human performance, such as sleeping, working environment, physical condition and mental state [6]. Here we regard the student stress level as an index of the student working efficiency. Two general quantification methods are recommended here. One is based on the human circadian rhythm, and the other is based on arousal theory.

Circadian rhythm is a general description of the physiological process of the human body, roughly in a cycle of 24 hours. Though it is believed as an endogenous variable, but still can be modulated by the environment, such as temperature and sunlight. Schmidt et al. [7] reviewed several experiments considering multiple factors, such as age, cognitive domain and test batteries. Carrier and Monk [8] reviewed task protocols, performance models and the correlation between circadian rhythm and endogenous factors, such as cortisol and melatonin. It is believed that circadian rhythm is closely related to sleep. A description of the neural activity resulting from sleep deprivation and a detailed discussion about the relations between circadian rhythm, sleep deprivation and performance can be found in [9]. 
Supposing human performance is dependent on time of day statistically, the working efficiency can be quantified with circadian rhythm data. Blatter and Cajochen [10] showed several set of experimental data which may show different types of the performance. Measures of the performance can be drawn from the data, such as an average index of the performance, or the normalized power of the curve in a specified time slot. It is undeniable that the circadian rhythm description based on performance tasks is very general without considering individual difference and other periodical physiological varieties, but to some degree, it is a way to quantify the performance during a day. A statistical circadian rhythm analysis with classification based on curve morphology can be found in [11].

It is widely known that stress can be embodied in several physiological indices, such as HRV, pupil diameter, body temperature, GSR, respiration rate and etc. A ratio of low frequency (LF) power and high frequency (HF) power computed from HRV can be regarded as an index of mental stress. Besides, electroencephalogram (EEG) alpha power density is also regarded as an index of arousal level in recent years [12]. Considering the individual difference, a normalized personal $\mathrm{LF} / \mathrm{HF}$ ratio or alpha power density can be used to describe the online arousal level.

\section{COURSE SCHEDULING ALGORITHM}

Course scheduling problem, or university course timetabling problem (UCTP), is a classic combinatorial problem with NP-hard, which means with the problem size growing, the time needed to find an optimal solution will increase exponentially. The basic problem of course scheduling is straightforward: to pair courses with time slots while considering two types of constraints. The first type of constraints are hard constraints which ensure the feasibility of the solution, such as classroom availability and capacity, time slot availability, etc. The second type of constraints are soft constraints, such as professors' preferred time slot, no consecutive classes for the same professor, etc.

Mathematically, the course scheduling problem is a zero-one programming problem. But owing to the NPcomplete feature, optimal solution(s) cannot be acquired in a polynomial time. Therefore, approximation algorithms are preferred over exact algorithms, such as dynamic programming and backtracking, in practice.

\subsection{Mental Fatigue Model}

After the quantification of the course difficulty and working efficiency, two vectors should be as follows: (i) Course difficulty: $\vec{D}=\left\{D_{l} \ldots D_{m}\right\}, m$ is the number of the total courses. It should be noted that if the same course occupies multiple time slots in a period, one day in default in this paper corresponding to the period of working efficiency measurement, it should be regarded as different courses;

(ii) Student stress in a time slot: $\vec{S}=\left\{S_{I} \ldots S_{n}\right\}, n$ is the number of time slots.

If the measurement is normalized, the value should be in $[0,1]$, and $[0,+\infty]$ if not.

According to the scheduling solution, the student stress for courses should be $\left\{\begin{array}{lll}S C_{1} & \ldots & S C_{m}\end{array}\right\}$. Considering the course capacity: $\vec{C}=\left\{\begin{array}{lll}C_{1} & \ldots & C_{m}\end{array}\right\}$, the total student stress can be expressed as

$$
T_{s}=\sum_{i=1}^{m}\left(C_{i} * D_{i} * S C_{i}\right)
$$

\subsection{Solution Generation}

Evolutionary algorithms (EAs) are widely used in solving course scheduling problem, such as genetic algorithm (GA), particle swarm optimization (PSO), simulated annealing (SA), etc. With different kinds of heuristic information, EAs are usually able to find suboptimal solutions within a reasonable time. The penalty method is often applied to involve two types of constraints into an objective function. Usually with a larger penalty parameter, the hard constraint can be satisfied first, which ensures the feasibility of a solution. Then the student stress and the other soft constraints, if involved, will be optimized.

Regarding the course scheduling problem as a multiobjective optimization problem is another means. In multi-objective optimization, the final set of solutions is termed as Pareto optimal solutions. In the solution set, no one is dominant over others for all objectives, i.e., no objective function can be improved without degrading other objective values. And along the Pareto frontier, all Pareto optimal solutions can be considered equally good. Supposing all solutions satisfy the hard constraint, how to make a trade-off between the student stress and the other soft constraints is up to the decision makers.

One resolution to generate a course schedule considering the student stress is to generate a set of solutions which satisfy the hard constraint, rank them by the student stress and choose the best one. Considering the course scheduling systems are widely applied in universities, this resolution is feasible.

Another way is integrating the student stress objective into the optimization algorithm objective function, with a penalty method or a multi-objective method. 


\section{CONCLUSIONS}

In order to increase the retention rate, a student stress constraint is involved in the course scheduling problem to reduce the student stress. Some future work still needs to be done as follows:

(i) Since the proposed method is based on empirical hypothesis without validation, an experiment is needed to check its validity;

(ii) Based on the Yerkes-Dodson Law, the relationship between performance and stress is nonlinear. Considering the ability distribution of all students, whether to reduce student stress in the proposed way is beneficial or not should be deliberated;

(iii) In practice, different courses in different time slots will change circadian rhythm curve. How to solve the course scheduling problem with considering the dynamical relationship between course and stress needs to be taken into consideration in the future work.

\section{Acknowledgements}

The author would like to acknowledge the StateSponsored Scholarship for Graduate Students from the China Scholarship Council.

\section{References}

[1] T.A. Nguyen and Y. Zeng, "A theoretical model of design creativity: nonlinear design dynamics and mental stresscreativity relation," J. Integr. Des. Process Sci., vol. 16, no. 3, pp. 65-88, 2012.

[2] C. Zhao, Y. Zhao, X. Tan, Y. Li, L. Luo and Y. Xiong, "Course evaluation method based on analytic hierarchy process," in: Y. Zhang (Ed.) Future Communication, Computing, Control and Management. Berlin Heidelberg: Springer, 2012. pp. 275-283. \{ISBN: 978-3-642-27313-1\}

[3] J.P. Caulkins, P.D. Larkey and J. Wei, Adjusting GPA to reflect course difficulty. H. John Heinz III School of Public Policy and Management, 1996.

[4] B. Cinaz, B. Arnrich, R. Marca and G. Tröster, "Monitoring of mental workload levels during an everyday life officework scenario," Personal Ubiquitous Comput., vol. 17, no. 2, pp. 229-239, 2013.

Available from

http:// dms.ife.ee.ethz.ch/index.php/attachments/single/838

[5] D.J. Mundfrom, Estimating course difficulty. Digital Repository @ Iowa State University, 1991, 249 pp.

[6] J.L. McLauglin, Stress, fatigue and workload: determining the combined affect on human performance. Florida: University of Central Florida Orlando, 2007, 530 pp.
[7] C. Schmidt, F. Collette, C. Cajochen and P. Peigneux, "A time to think: circadian rhythms in human cognition," Cognitive Neuropsychology, vol. 24, no. 7, pp. 755-789, 2007.

[8] J. Carrier and T.H. Monk, "Circadian rhythms of performance: new trends," Chronobiology International, vol. 17, no. 6, pp. 719-732, 2000.

[9] N. Goel, M. Basner, H. Rao and D.F. Dinges, "Circadian rhythms, sleep deprivation, and human performance," Progress in molecular biology and translational science, vol. 119, pp. 155-190, 2013.

[10] K. Blatter and C. Cajochen, "Circadian rhythms in cognitive performance: Methodological constraints, protocols, theoretical underpinnings," Physiology \& Behavior, vol. 90, no. 2-3, pp. 196-208, 2007.

[11] D.S. Minors and J.M. Waterhouse, "Mathematical and statistical analysis of circadian rhythms," Psychoneuroendocrinology, vol. 13, no. 6, pp. 443-464, 1988.

[12] D. Hagemann, J. Hewig, C. Walter, A. Schankin, D. Danner and E. Naumann, "Positive evidence for Eysenck's arousal hypothesis: A combined EEG and MRI study with multiple measurement occasions," Personality and Individual Differences, vol. 47, no. 7, pp. 717-721, 2009. 